\title{
Neuroendocrine Tumor Biomarkers: Current Status and Perspectives
}

\author{
Irvin M. Modlin ${ }^{\mathrm{a}, \mathrm{c}}$ Kjell Oberg ${ }^{\mathrm{d}} \quad$ Andrew Taylor $^{\mathrm{a}} \quad$ Ignat Drozdov ${ }^{\mathrm{a}}$ \\ Lisa Bodei ${ }^{b}$, Mark Kidd $^{\text {b }}$ \\ ${ }^{a}$ Wren Laboratories, Branford, Conn., and Departments of ${ }^{b}$ Surgery and ${ }^{\complement}$ Gastroenterological Surgery, Yale School \\ of Medicine, Yale University, New Haven, Conn., USA; 'Department of Endocrine Oncology, University Hospital,

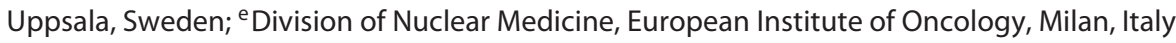

\author{
Key Words \\ Biomarker · Chromogranin A · Circulating tumor cells . \\ Multianalyte assays · Algorithmic analyses · Monoanalytes . \\ MicroRNA · Neuroendocrine tumor · Pancreastatin . \\ Serotonin - Transcript
}

\begin{abstract}
The identification of accurate harbingers of disease status and therapeutic efficacy are critical requirements in precise diagnosis and effective management. Initially, tissue analysis was regarded as ideal but invasive strategies represent risk compared with peripheral blood sampling. Thus far, most biomarkers, whether in tissue or blood/urine, have been single analytes with varying degrees of sensitivity and specificity. Some analytes have not exhibited robust metrics or have lacked methodological rigor. Neuroendocrine disease represents an area of dire biomarker paucity since the individual biomarkers (gastrin, insulin, etc.) are not widely applicable to the diverse types of neuroendocrine neoplasia. Broad-spectrum markers such as chromogranin A have limitations in sensitivity, specificity and reproducibility. Monoanalytes cannot define the multiple variables (proliferation, metabolic activity, invasive potential, metastatic propensity) that constitute tumor growth. The restricted status of the neuroendocrine neoplasia field has resulted in a lack of comprehensive knowledge of the molecular and cellular biology
\end{abstract}

of the disease, with tardy application of innovative technology. This overview examines limitations in current practice and describes contemporary viable strategies under evaluation, including the identification of novel analytes (gene transcripts, microRNA), circulating tumor cells and metabolic imaging agents that identify disease. Novel requirements are necessary to develop biomathematical algorithms for synchronous calibration of multiple molecular markers and predictive nomograms that interface biological variables to delineate disease progress or treatment efficacy. Optimally, the application of novel techniques and amalgamations of multianalyte assessment will provide a personalized molecular disease signature extrapolative of neuroendocrine neoplasia status and likelihood of progression and predictive of therapeutic opportunity.

(c) 2014 S. Karger AG, Basel

\section{Introduction}

This study serves to provide a general background on biomarkers and to describe their current and past usage and shortcomings in neuroendocrine tumors (NETs). It serves to deliver a broader view with respect to describing the changing methodology of biomarker development and defining the relevance of this to treatment and detection. Finally, it presents current work within this context

\section{KARGER}

E-Mail karger@karger.com

www.karger.com/nen (c) 2014 S. Karger AG, Basel

0028-3835/14/1004-0265\$39.50/0
Irvin M. Modlin

Yale School of Medicine, Yale University

333 Cedar Street, PO Box 208062

New Haven, CT 06520-8062 (USA)

E-Mail imodlin@optonline.net 
and discusses future and possible developments in the field.

Future progress in the treatment of advanced NET disease requires a fundamental understanding of the underlying causes and mechanisms of neoplasia. Irrespective of such requirements, the need for early detection is paramount, since the discrete ideologies of detection and treatment are mutually reconcilable. The development of strategies to facilitate early detection may be regarded as either an agnostic process or pantheistic depending on the methodologies employed. The assessment of marker 'value' requires the application of a variety of techniques, including biopsy, imaging, cell collection, or measurement of a bioanalyte (biomarker). Each may singly, or ideally in concert, be correlated with the presence or level of a disease state or assessment of the efficacy of diverse therapies (surgery, pharmacological agents, radiation, tissue ablation).

Since the inefficacy and costs of late therapy are palpably clear, biomarkers represent a high yielding facet of medicine at a scientific, clinical and economic level as well as contributing to the improvement of survival and quality of life. Their deliverables include earlier diagnosis, identification of residual disease, minimal disease detection, support of other complex investigative modalities, and demonstration of failure/efficacy of therapy. Overall, the biomarker compendium includes the identification of novel chemicals expressed in complex diseases, the mechanistic development of more effective tests, clinical deployment, informatics, and the subsequent regulation of tests. The advent of discovery-based approaches such as array-based detection of gene expression and proteomic approaches using mass spectrometry has identified and will predictably identify a plethora of candidate cancer biomarkers [1].

Many new biomarkers are targeted toward treatment selection, and most require tumor biopsy samples. Thus, biomarker assays that predict tumor aggressiveness have been developed for breast and colon cancers (Oncotype DX and MammaPrint) and are used clinically to select patients for adjuvant chemotherapy in early-stage disease. In lung adenocarcinomas, EGFR sequencing can identify mutations in 5\% that will respond to the EGFR inhibitor gefitinib (Iressa) [2]. Similarly, in individuals with gastrointestinal stromal tumors treated with imatinib (Gleevec), a therapeutic response can be identified within days using ${ }^{18} \mathrm{~F}-\mathrm{FDG}$ PET imaging [3]. In NET disease an analogous protocol, somatostatin receptor imaging (and immunohistochemistry), is useful in the prediction of tumor response to somatostatin analogs and/or targeted peptide receptor radiotherapy [4]. The use of biomarkers or molecular imaging, either alone or in concert, can therefore indicate treatment efficacy, enabling rapid cessation of ineffectual therapies in favor of other potentially effective treatments.

Based upon the rapidly advancing and critical context of this component of medicine, defining biomarkers, demonstrating their utility and assessing their potential in relation to NETs are critical requirements necessary to advance the management strategy of this neoplastic group. To date, biomarkers for this class of tumors have been limited in their sensitivity, specificity and prognostic metrics [5-7].

\section{What Constitutes a Biomarker and How Can It Be Utilized?}

Biological markers (biomarkers) are 'cellular, biochemical or molecular alterations that are measurable in biological media such as human tissues, cells or fluids' [8]. This definition has been expanded to include biological characteristics that can be objectively measured and evaluated as an indicator of normal biological processes, pathogenic processes or pharmacological responses to a therapeutic intervention. Overall, biomarkers include tools and technologies that can facilitate the prediction, cause, diagnosis, progression, regression, or outcome of treatment of disease. Such information may be derived from a variety of sources including, but not limited to, tissue, body fluids and imaging. Overall, the most usable biomarkers represent cell surface or secreted proteins. Candidate biomarkers expressed in the nucleus or cytoplasm, therefore, provide a limitation since these are generally not accessible to clinical assays.

\section{NIH Definition of Biomarkers}

The NIH proposed a biomarker classification system based on validation and clinical usage (fig. 1) [9]. In brief, it comprises the following: type 0 markers: 'indicators of the natural history of disease' - these markers correlate with diagnosis, prognosis and outcome but their relationship with the disease can be superficial or nebulous; type I markers: 'capture the effects of an intervention in accordance with the mechanism of action of the drug' - a type I biomarker reflects the general efficacy of treatment, without necessarily being linked to the specific mechanism being exploited, and type II markers: those that are used as surrogates for clinical endpoints, the term 'end- 


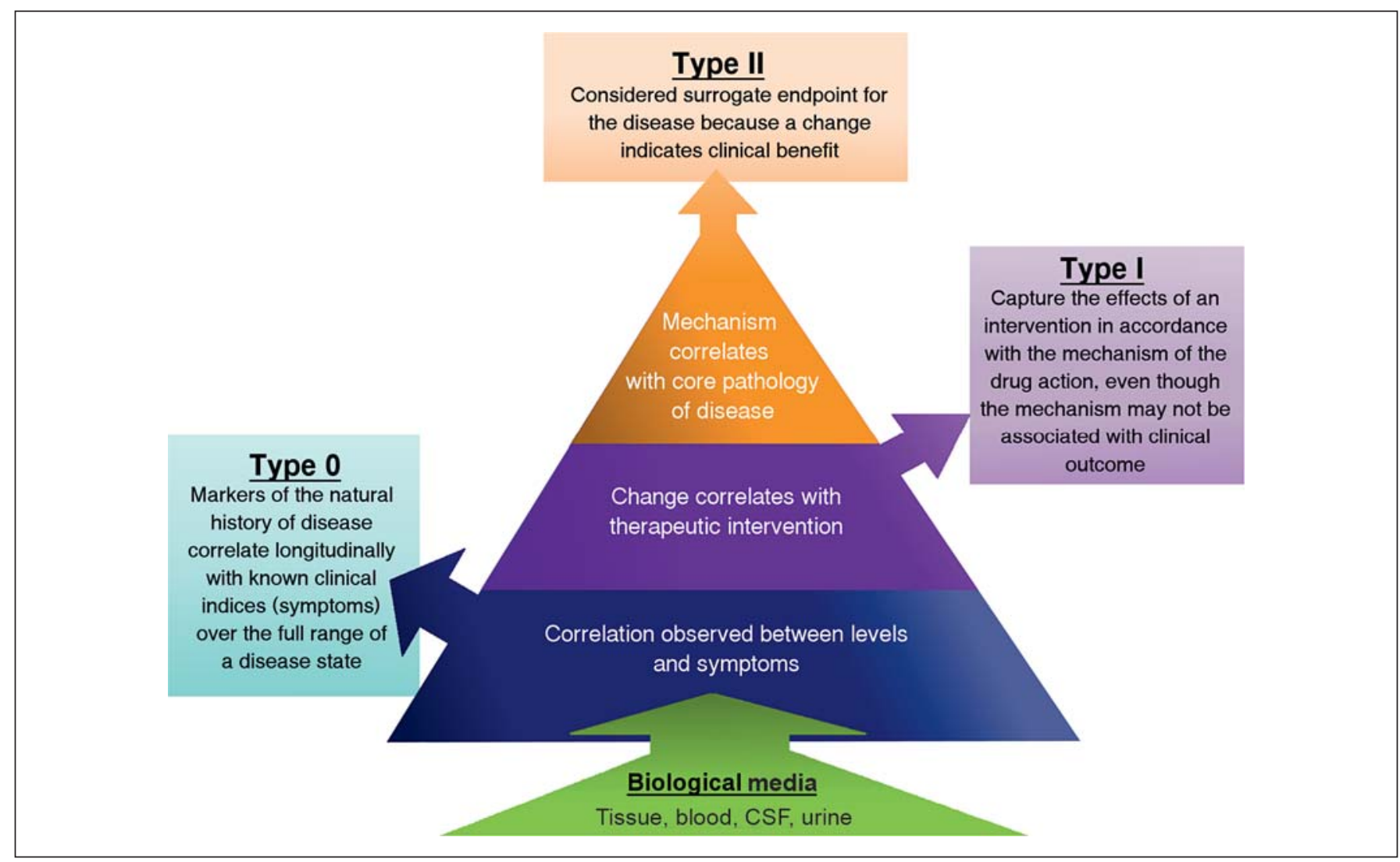

Fig. 1. The NIH biomarker classification proposal [9] designates 3 different categories. Type 0 correlates with diagnosis, prognosis and outcome, type I reflects the general efficacy of treatment and type II is used as a surrogate for clinical endpoints. The relationship of each type with disease ranges from tenuous (type 0 ) to being mechanistically linked to the disease (type II). point' reflecting patient health, functionality and survival (fig. 1). This generates significant difficulties for the development of NET biomarkers since the disease comprises a heterogeneous group of tumors which exhibit a spectrum of pathological and clinical expression.

\section{General Limitations of Biomarkers}

\section{Economic and Commercial Considerations}

Economic considerations have, to some extent, inhibited the development of cancer detection biomarker development. Many of the diagnostic and prognostic markers are in the public domain and lack intellectual property protection; thus companies have avoided developing clinical tests without such protection. Even when an assay is protected, companies face challenges in developing a clinical-grade assay with performance characteristics in reproducibility and accuracy that exceed academic research standards. FDA approval of a clinical diagnostic requires scrutiny of the assay and demonstration of effectiveness in altering clinical decision making such that a favorable health impact can be demonstrated. In the last decade, the FDA has approved few biomarkers.

\section{Meeting Acceptable Scientific Criteria}

Optimally, a cancer detection biomarker should be found uniquely in the malignant tissue of interest and should generate a positive signal that can be measured without confounding 'noise' from normal tissues or other nonmalignant pathologies. Although gene expression profiling has been highly touted as a future strategy, it identifies thousands of genes expressed at higher levels in malignant compared with benign tissues, but only limited numbers of transcripts or proteins have been identified that are uniquely elevated in cancer [10]. Optimization, therefore, requires the identification of multiple markers and the development of mathematically weighted algorithms to identify abnormal quotients reflective of a specific neoplasia. 


\section{Specific Biomarker Problems}

False Positives. Limiting false-positive tests may be achieved through screening high-risk populations with identified germ-line 'high-risk' alleles [11-13]. Assays of SNPs, either singly or in groups, have been of marginal value to risk assessment. Important exceptions include BRCA1, BRCA2, VHL, and TP53, amongst a handful of other germline mutations. Hereditary cancer syndromes constitute only a small fraction $(<5 \%)$ of NET patients (multiple endocrine neoplasia I and II and von HippelLindau disease) and usually occur in clinically recognizable familial clusters. Thus, biomarkers that assay either rare or low-risk alleles are not useful for screening.

Identification of Somatic Mutations. Acquired alterations in cancer DNA have the potential for detection since they are unique to the cancer. In serous ovarian cancer, $96 \%$ possess a mutated TP53 gene [14]. Point mutations in specific oncogenes such as KRAS have been identified in colon cancer [15]. Cancer-specific hypermethylation of cytidine residues in CpG islands is common [16-19]. Fusion transcripts, such as TMPRSS2-ERG in prostate cancer and identifiable in urine, are examples of potential cancer-specific biomarkers [20]. The identification of somatic mutations in NETs has proved of limited value [21].

Cancer Genome Analysis. In almost all malignancies, assays of a single structural alteration or mutation will identify only a fraction of prevalent cancers, necessitating the need to develop multiplex assays to interrogate entire pathways or many chromosome loci [14]. An additional issue is that such strategies must also be able to detect mutated sequences against a background of wild-type sequences (naturally occurring SNPs) that may be present in any clinical sample (e.g. blood or urine).

\section{The Utility of Biomarkers for Screening}

General Considerations. The behavior of a screening biomarker, especially in the context of NETs, is influenced by how frequently the malignancy occurs in a population of individuals. Because the incidence rates for NETs are low, any screening tool must display relatively high sensitivity (the portion of cancer cases that have a positive test) and specificity (the portion of individuals without cancer that have a negative test). Tests with poor performance characteristics can miss cases (false negatives) or identify individuals as harboring the disease when they do not (false positives). False-negative tests have obvious consequences. False-positive tests will lead to expensive and often invasive ancillary tests. Since the potential for financial and personal harm is substantial, tests must exhibit high sensitivities and specificities.

Specificity. There is minimal knowledge with regard to the biological sequence that enables transformed cells to become malignant and ultimately lethal. This limitation has major implications for biomarker performance since, to have utility, a screening strategy must identify malignant cells that are destined to grow, metastasize and cause death. Since neoplasia, in addition to intrinsic proliferation, reflects a multiplicity of other tissue components and diverse cell systems, features of the host, including the innate immune response, interactions between neoplastic cells and proximate normal tissue (and/or stochastic factors that may not be captured by any biomarker), are important in the progression of early lesions [22].

An issue of continued focus is that neoplastic lesions comprise heterogeneous populations of malignant cells, including small populations of cancer stem cells that have been proposed as a source of metastatic cells $[23,24]$. A single biomarker may not capture the natural history of the disease. Many tumors take years or even decades before progression to lethality. There may thus be a substantial time frame for the detection and eradication of transformed cells. In small intestinal NETs where the majority of individuals exhibit 5-year survival figures of greater than $\sim 80 \%$, there is substantial time to detect alteration in disease status [25].

A key unmet need is the necessity to predict future neoplastic behavior (or prognosis) since the alternative is overtreatment (in the case of gastric NETs, antrectomy or in rectal NETs, anterior resection) or repetitive imaging lacking in adequate discriminant ability to identify early progression [4]. Alternatively, developing the ability to predict progress or metastasis provides an opportunity to increase the window of effective therapeutic opportunity, which is a critical issue in NET disease. The successful development of a biomarker for NET detection must be linked to prognostic biomarker discovery to avoid overtreatment of clinically indolent neoplasia or late treatment of advanced lesions.

\section{Measurement and Metrics}

While the term 'accuracy' is used as shorthand to describe biomarker tests, a preferable depiction of the scientific 'power' of a biomarker is sensitivity and specificity. While their individual importance may be decided by purpose, they are medically valuable as quantifiers since they 
represent these two most important aspects. Sensitivity is the rate of true positives, or the percentage of diseased individuals that the test detects. Specificity is its counterpart, the rate of true negatives, or the percentage of individuals without the disease who are truly negative. Low sensitivity results in a high false-negative rate, while low specificity results in numerous false positives. If either quantifier is too low, the effective power of a test diminishes in a manner not expressed adequately by accuracy. An elegant graphical representation of the balance between sensitivity and specificity is the receiver-operating curve, in which 'each point represents a conditional probability of a test result from a random diseased subject exceeding that from a random nondiseased subject' [26]. This creates a portrait where the area under the curve (AUC) represents a convenient graphical ideogram of the diagnostic power of the test. Biomarkers can effectively be compared in the same graph by the magnitude of their AUCs. The higher the AUC, the better a biomarker is performing; in general, AUCs should be greater than 0.8 (moderately accurate) [27].

If the purpose of the test is screening for a potentially life-threatening disease and the patient is symptomatic, a test with high sensitivity is preferable to potentially overlooking a dangerous condition. This perspective is referred to as SnOUT (High Sensitivity Rules Out). Its counterpart is SpIN (High Specificity Rules In). A test with high specificity is most effectively used to accurately confirm a diagnosis with a high level of accuracy when the cost (impact) of incorrectly assuming disease presence would be high, e.g. if positivity determined the need for investigative surgery [28]. In order to further delineate the clinical relevance of a biomarker, positive and negative predictive values require assessment. These terms define the chance that an individual test result is reliable, whether positive or negative.

\section{The Need for NET Biomarkers}

The progress and challenges presented by biomarkers assume a special relevance in the study of NETs. From the point of view of biomarker targets, such class of neoplasia represents the 'perfect storm'. NETs are heterogeneous and variable due to their diverse anatomical and cellular origins and are subsequently protean in their production of measurable bioactive products and diverse symptomatology as well as tumor behavior. They are of relatively low incidence (estimated overall 5.25 cases per 100,000 persons in 2005) [29] but exhibit an increasing frequency and prevalence, the most common being gastroentero-
Table 1. Current NET monoanalyte biomarkers

\begin{tabular}{|c|c|c|c|c|}
\hline Biomarker & Type & Sensitivity & Specificity & References \\
\hline $\mathrm{CgA}$ & $0-\mathrm{II}$ & $43-100 \%$ & $10-96 \%$ & $\begin{array}{l}{[39,52-54,} \\
89-91]\end{array}$ \\
\hline u5-HIAA & $0-\mathrm{II}$ & $35 \%$ & up to $100 \%$ & {$[65]$} \\
\hline Substance P & 0 & $32 \%$ & $85 \%$ & {$[67]$} \\
\hline Pancreatic polypeptide & 0 & $50-80 \%$ & no data & [92] \\
\hline Pancreastatin & $\mathrm{I}$ & $64 \%$ & $58-100 \%$ & {$[53,54,91]$} \\
\hline NSE & $\mathrm{I}$ & $33 \%$ & up to $100 \%$ & {$[65]$} \\
\hline $\mathrm{NKA}^{1}$ & $\mathrm{I}$ & $88 \%$ & no data & {$[57]$} \\
\hline $\mathrm{CgB}$ & I & $99 \%$ & no data & [53] \\
\hline ProGRP & I & $99 \%$ & $43 \%$ & {$[74]$} \\
\hline NT-BNP ${ }^{2}$ & II & $87 \%$ & $80 \%$ & {$[71]$} \\
\hline $\mathrm{CTGF}^{3}$ & II & $88 \%$ & $69 \%$ & {$[73]$} \\
\hline
\end{tabular}

u5-HIAA = Urinary 5-HIAA; ProGRP = progastrin-releasing peptide; NT-BNP $=\mathrm{N}$-terminal brain natriuretic peptide.

${ }^{1}$ Midgut NETs. ${ }^{2}$ In carcinoid heart disease. ${ }^{3}$ For right-ventricular dysfunction.

pancreatic and bronchopulmonary NETs. Since they are often indolent and their symptomatology mundane, detection is often much delayed. As a consequence, they are often diagnosed at a late stage with metastatic progression. Since treatment efficacy is dependent on an early diagnosis, screening and detection are a high priority [30]. Therefore, the identification and demonstration of an effective biomarker set in NET disease constitutes a high priority.

\section{Traditional Biomarkers: The Single-Analyte Approach}

Single-analyte biomarker development is based on the identification of straightforward, usually ratio-based correlations of, for example, a protein, single gene or RNA whose level is representative of a disease and can be judged based upon the development of a relative scale. To further complicate the matter, there is no overarching standardization of concentrations, even within commercial tests for accepted biomarkers, so the method provided by each manufacturer can acutely affect the result. This can produce a range of values for sensitivities and specificities across a given biomarker, as evidenced in the large diversity of chromogranin A (CgA; table 1) [31]. The failure to achieve standardization substantially limits the proper evaluation of the test and is especially challenging at a clinical level since reproducibility varies [32]. 


\section{Multianalyte Assays with Algorithm Analyses}

To improve sensitivity and specificity in screening, a common technique is to measure an additional biomarker that addresses the deficits in the other. These tests, called multianalyte assays with algorithmic analyses (MAAAs), based on correlating and normalizing multiple sets of variables, are increasing in usage and show applicability in many diseases. FibroSure (FibroTest), for example, is a six-panel blood test for liver damage which has become widely used for hepatitis $\mathrm{C}$ diagnosis and detection and can replace liver biopsy [33, 34]. MammaPrint, a 70-gene assay test for metastatic breast cancer, was FDA approved in 2007. It is one of three commonly used, commercially available tests which also include Oncotype DX and Mammostrat. Multianalyte testing has also shown promise in longitudinal screening: chronic lymphocytic leukemia and ovarian cancer have been shown to be detectable with a multiple gene test [35]. In the case of leukemia, surveillance genes are detectable 10 years prior to presentation [36].

The development and application of multianalyte biomarkers are significantly more interdisciplinary than single analytes, necessitating a comprehensive statistical and systems approach for the identification and utility of new panels. Trends within multiple-variable relationships can only be detected by the use of considerable mathematical power and complex analytic techniques. Currently, the rate-limiting step of MAAA development is regulation. To some extent this reflects the current paradigm with regard to the utility of biomarkers being geared towards the much simpler relationships of the single-analyte tests [37].

\section{Current NET Biomarkers: Monoanalytes}

While numerous single-analyte type 0 markers have been evaluated in NETs, there is a dearth of clinically useful type II tests. The methodology for selecting biomarker candidates thus far has focused on the symptoms of the disease (carcinoid syndrome) and the secretory products of different neuroendocrine cells.

A prominent biomarker of this kind is represented by $\mathrm{CgA}$. The granin family of peptides is a unique secretory family of water-soluble acidic glycoproteins that are expressed in excretory vessels in neurons and endocrine cells. The plasma (or serum) measurement of $\mathrm{CgA}$ is the result of this line of investigation. It is predictable that pancreastatin, of which CgA is a precursor, has also been

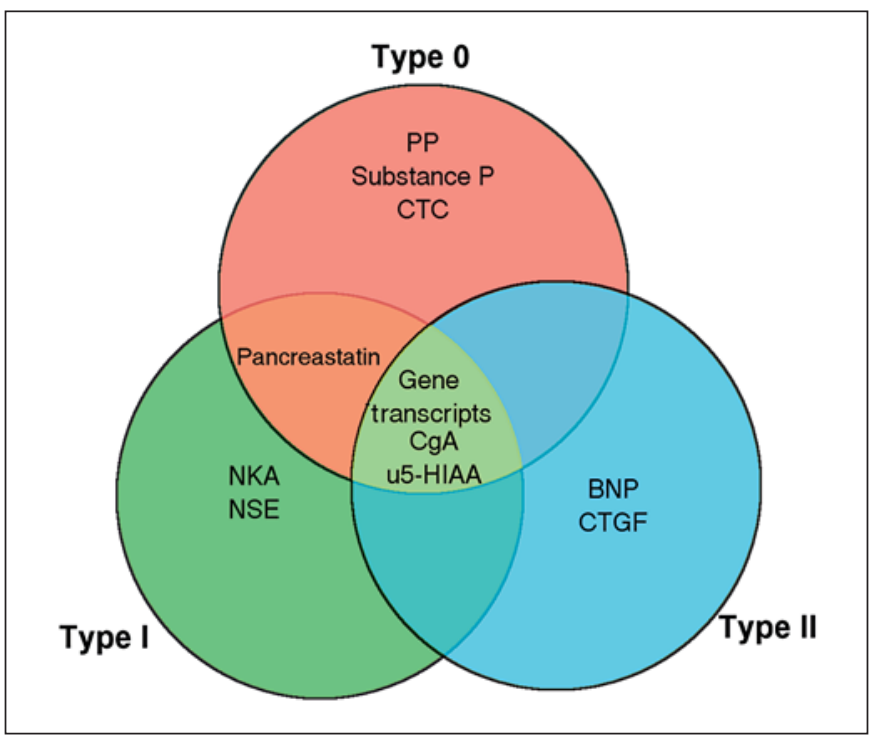

Fig. 2. Distribution of current NET biomarkers based upon the $\mathrm{NIH}$ classification system [9]. Type 0 markers include pancreatic polypeptide, substance P and CTCs. Type I markers include NKA, NSE, progastrin-releasing peptide, $\mathrm{CgB}$ and pancreastatin. Type II biomarkers include brain natriuretic peptide and CTGF - these are, however, limited to specific patient populations with serotonin-mediated cardiac disease. The NET gene transcript test, CgA and 5-HIAA reflect all 3 biomarker types. $\mathrm{BNP}=$ Brain natriuretic peptide; $\mathrm{PP}=$ pancreatic polypeptide; Pro-GRP = progastrin-releasing peptide; u5-HIAA = urinary 5-HIAA.

assessed as a candidate biomarker. Serotonin and its final secreted product, 5-hydroxyindoleacetic acid (5HIAA, particularly urine-collected) are also commonly tested as type I and II biomarkers (fig. 2). Other peptides and amines that occur in neuroendocrine cells, including neuron-specific enolase (NSE) and neurokinin A (NKA), have also been considered, usually as type 0 biomarkers. These and several other previously investigated biomarkers are discussed below in more detail, and a table is provided comparing their sensitivities and specificities (table 1).

Chromogranin A. To date, the most widely accepted test for NETs has been CgA. An increased CgA level is 'generally considered' to be sensitive, $\sim 60-90 \%$, and accurate once an NET has been identified [38]; it is, however, an ineffective first-line diagnostic [39]. Measurements are usually nonspecific (10-35\% specificity) [4042]. CgA suffers from all of the problems inherent in single-analyte tests, i.e. it is expressed in healthy tissue and is commonly elevated in other neoplasia e.g. pancreatic, small cell lung neoplasia and prostate carcinomas [43] as well as in cardiac and inflammatory diseases [44] 
and by renal failure [45]. Background levels are variable in different populations and are adversely affected by food ingestion [46] and the use of proton pump inhibitor (PPI) administration [47], which is commonly used, particularly in older populations $[48,49]$. Furthermore, there is a high level of variability between CgA kits, resulting in uncertain standardization (in one kit it was noted that the manufacturer listed the final result of the concentration calculation in 'units/liter') [31]. Overall, individual CgA immunoassays tend to be poorly correlated with each other, making interassay assessments difficult [47].

To compensate for these limitations, $\mathrm{CgA}$ is frequently used with a high cut-offlevel (one study used 84-87 U/1 to obtain a specificity of $95 \%$, resulting in sensitivity of $55 \%$, in comparison with healthy, low-CgA selected controls [50]). While CgA is correlated with tumor load, levels tend to be highest in metastatic cancer, particularly in the liver [51], so its efficacy in early detection is questionable. Despite this, CgA has been used to confirm a diagnosis and, once a baseline level is established, can provide some insight into tumor burden as well as act as a surrogate endpoint [52]. These attributes suggest measurement of a protein that can be of limited utility as a type II biomarker but with caveats.

Pancreastatin. Pancreastatin is gaining clinical significance as a smaller, leaner, more specific polypeptide product of CgA. It has been reported to be variously elevated in $58-81 \%$ of NETs $[53,54]$. Despite being a derivative of CgA, pancreastatin levels are not affected by PPI use [55] and, compared with its larger, less predictable parent molecule, has been proposed as a more stable measurement of tumor activity [56]. Although pancreastatin showed a greater sensitivity and specificity than $\mathrm{CgA}$ in a comparative study [57], this merely offers to improve upon the most troublesome aspects of $\mathrm{CgA}$ without solving the underlying difficulties of complex expression in NETs. This is underscored by observations that pancreastatin does not correlate with tumor location, is not associated with tumor functionality and may not correlate with tumor aggressiveness or patient survival [54]. It can be affected by medications or food ingestion e.g. glucose may alter test results [58], and levels have been reported to be elevated in diabetics [59] as well as in hyperparathyroidism [60]. Some investigators consider that different conformations of the peptide occur and 'masking' may render assay interpretation complex and controversial [61].

Other Granins. Chromogranin B (CgB) and chromogranin $\mathrm{C}(\mathrm{CgC})$ have been examined but their reliability in NETs is significantly lower than CgA. In a comparative study of 44 patients, CgA was expressed in nearly $100 \%$,
$\mathrm{CgB}$ was present in $88 \%$ and $\mathrm{CgC}$ was only present in $6 \%$. A commercial assay is available for circulating $\mathrm{CgB}$ which, unlike that used for CgA, is not affected by PPIs or impaired kidney function [62].

Neuron-Specific Enolase. NSE is an enolase present in neurons and neuroendocrine cells and can be indicative of tumors derived from these cell types. NSE cannot differentiate between different subtypes of NETs, but elevated NSE levels may identify poorly differentiated tumors. NSE is no longer commonly used in clinical practice as it offers no particular benefit over $\mathrm{CgA}$ and performs worse [63].

Serotonin and 5-HIAA (urinary). Serotonin and its metabolite 5-HIAA are the second most thoroughly explored NET biomarkers due to their initial designation as the gold standard. Used because serotonin is secreted in large amounts in some patients, it previously required repeated urinary collection and measurement. More recently, plasma measurements have simplified the process and proven as accurate as urine analysis [64]. However, while 5-HIAA showed a high specificity of $\sim 100 \%$, low sensitivity ( $35 \%$ [65]) due to diverse expression diminished its performance. Furthermore, 5-HIAA has only a tenuous connection to severity, often showing little to no correlation [66]. From a functional perspective, this has restricted its use to screening and diagnosis, although it has been used as a type II marker of disease course.

\section{Miscellaneous NET Biomarkers}

Tachykinins. These are neuropeptides involved in nociception and smooth muscle contraction. NKA and the related substance $\mathrm{P}$ have been used as circulating markers for carcinoid tumors. NKA has been identified as having some prognostic value and correlates strongly with poor outcomes in midgut tumors [57]. Crucially, its significance is that it has been considered to signal therapeutic responses to somatostatin analogs, the most widely used symptomatic treatment of NETs. While NKA may have some utility for prediction in response to treatment as a type I marker and as an independent predictor of survival, substance $\mathrm{P}$ has limited applicability given its poor sensitivity without high specificity [67].

Pancreatic Polypeptide. This is produced by the pancreas and the colon and is considered to play a role in the autoregulation of secretion. It is not integral to any known aspect of NETs but has been used to limited effect as a nonspecific NET marker [68]. While it can detect islet cell tumors, in-depth assessment has revealed it to be of lim- 
ited clinical utility [69]. Combining measurements of CgA with pancreatic polypeptide for detecting gastroenteropancreatic NETs was determined to be of no added clinical utility [70].

N-Terminal Probrain Natriuretic Peptide. This has been proposed as an indicator of left-ventricular heart disease. This has rendered it a source of interest for carcinoid heart disease and as an indicator of prognosis. For predicting carcinoid heart disease in NETs, it has a high sensitivity and specificity ( 87 and $80 \%$, respectively). It also correlates with patient survival [71].

Connective Tissue Growth Factor. Connective tissue growth factor (CTGF) has acceptable metrics (>80\% accuracy and sensitivity) for heart disease [72]. In NETs, elevated CTGF is an independent predictor of reduced right-ventricular function, with $88 \%$ sensitivity and $69 \%$ specificity for its detection [73].

Progastrin-Releasing Peptide. Progastrin-releasing peptide has been proposed as a marker for primary lung NETs. It has been reported in one study to have a specificity of $99 \%$ and a sensitivity of $43 \%$ [74]. Expression was also strongly associated with survival; specifically, high levels (>90 ng/l) were negatively correlated with outcome (hazard ratio $=3.43$ ).

These overall disappointing investigations reflect the difficulty of identifying and utilizing biomarkers without first defining core mechanistic disease processes. Under such circumstances, the outcome is biomarkers that are incidental and whose relationship to the disease is nebulous and often at best representative as correlatable epiphenomena.

\section{Circulating Tumor Cells}

The detection of circulating tumor cells (CTCs) is based on technology that captures the cellular expression of the epithelial cell adhesion molecule marker. In NET studies, $43 \%$ of midgut and $21 \%$ of pancreatic NETs had detectable CTCs. No or low correlations were noted with Ki-67 and serum CgA, respectively [75]. In a subsequent study [76], $\sim 50 \%$ of patients were noted to have $\geq 1$ CTC. CTC numbers were increased when the liver burden was $>25 \%$ and weak correlations were noted with grade and CgA levels. No association was evident between somatostatin therapy and CTCs or between therapy and the number of CTCs. Large studies in epithelial-derived tumors (metastatic breast cancer [77], colorectal cancer [78], prostate cancer [79]) have reported a role for CTCs as an independent predictor of progression-free and over- all survival. Such data have not been convincingly reported with NET-CTC studies. The low number of patients with detectable CTCs in NETs, coupled with heterogeneity in epithelial marker expression as well as the absence of significant relationships with therapeutic response, has been disappointing. While the technology and concept have an attractive value proposition, the CTC technology platform does not indicate that it can be used in its current form as an effective biomarker for NETs.

\section{MicroRNAs}

MicroRNAs (miRNAs) are a class of small (19-25 nucleotides) noncoding RNAs that function as posttranscriptional regulators in diverse disease processes. Given their dysregulation in neoplasia, miRNAs are considered to have potential as both biomarkers and therapeutic agents [80]. The global miRNA profiles of pancreatic and small intestinal NETs [81-84] are distinguished by nonoverlapping expression in each NET type. In pancreatic NETs, an upregulation of miR-103 and miR-107 was identified [81], and miR-21 overexpression was associated with both a high Ki-67 proliferation index and liver metastases [81]. A second study determined that the expression of miR-642 correlated with the Ki-67 index while miR-210 was associated with metastatic disease [82]. At a serum level, miR-193b was upregulated in both tumor tissue and serum, although overall a low correlation between miRNA expression levels in serum and tissue was evident. In a separate study, The downregulation of serum miR-1290 was identified to discriminate pancreatic NETs from adenocarcinomas [83]. Other significantly downregulated serum miRNAs in pancreatic NETs were miR-584, miR1285, miR-550-002410, and miR-1825 [83]. In small intestinal NETs, five miRNAs (miR-96, miR-182, miR-183, miR-196, and miR-200) were upregulated during tumor progression, whereas four (miR-31, miR-129-5p, miR133a, and miR-215) were significantly downregulated [84]. No peer-reviewed data exist regarding the expression of these potential biomarkers in serum. In 2013, the American Association for Clinical Chemistry [85] noted that the detection and quantification of miRNAs were 'neither robust, rapid, simple, accurate, reproducible nor inexpensive' and 'low correlations are noted between either different platforms or from the same platform using reagents from different vendors'. Data normalization is also regarded as problematic. Overall, the lack of standardization and implementation of normalization 
Fig. 3. Assessment of the utility of current NET biomarkers and imaging based on published use and performance metrics. miRNA assessment is in an early phase with no rigorous utility or metrics available. CTC measurement exhibits moderate metrics but has not been validated as a specific NET biomarker. Substance P, NSE, pancreatic polypeptide, NKA, pancreastatin, CTGF and brain natriuretic peptide all display variable performance metrics. Their overall utility is weak and is based on examination of specific NET disease subsets. Biomarkers including urinary 5-HIAA, CgA and gene transcripts have moderate utility but the metrics of the former vary substantially. For comparison with biomarkers in use, imaging data derived from CAT scan, MRI, somatostatin receptor scintigraphy, FDG and ${ }^{68}$ gallium PET are included. All imaging techniques have high utility but there is a range in metrics from FDG to ${ }^{68}$ gallium PET. BNP = Brain natriuretic peptide; $\mathrm{CT}=\mathrm{CAT}$ scan; $\mathrm{Ga}-\mathrm{PET}={ }^{68}$ gallium PET; $\mathrm{PP}=$ pancreatic polypeptide; Pro-GRP = progastrin-releasing peptide; $S R S=$ somatostatin receptor scintigraphy; u5-HIAA = urinary 5-HIAA.

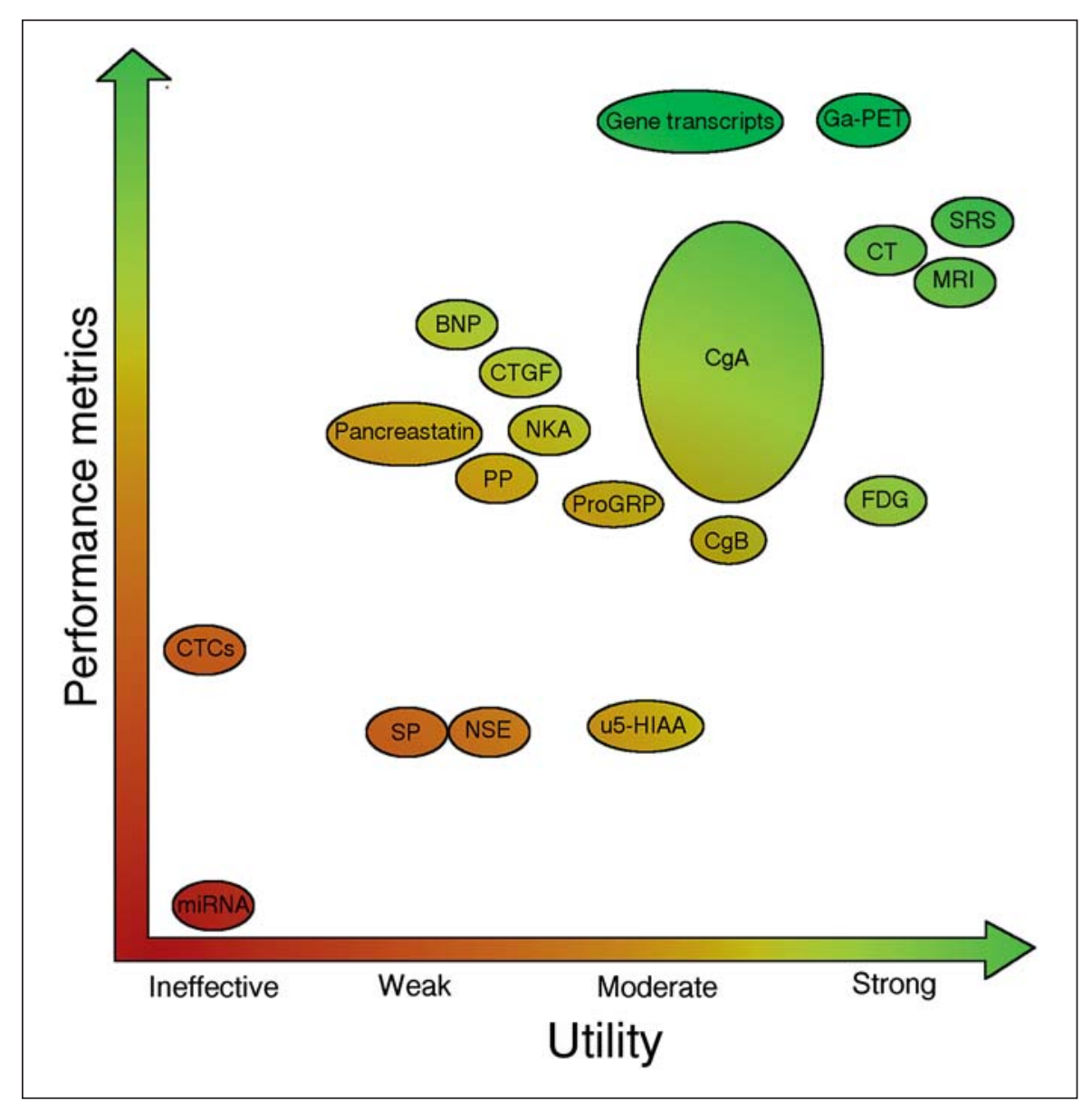

methods makes the evaluation of these targets as biomarkers and their clinical application challenging (fig. 3).

\section{NET Multianalyte Algorithmic Tests (MAAAs)}

It was noted by the NCI Neuroendocrine Tumor Summit Conference (2008) [86] that a substantial unmet need was the necessity to identify and develop biomarkers for the prediction, diagnosis, prognosis, and classification of NETs and to evaluate these markers as a response to treatment. Given the multiple interactive pathological and biochemical pathways in these tumors, monoanalyte markers fail to adequately represent the intricacies of NET disease. The molecular events that first precede detection, and then dictate prognosis and response to treatment, are complex and require a highly dimensionalized systems approach to map the disease in a clinically meaningful way. Current developmental technologies provide the ability to build multitier, high-content analyses that facilitate greater collection of data and subsequently provide insight into the diverse molecular components that comprise disease development. The simultaneous measurement of individual markers representative of multiple pathways allows delineation of a multidimensional picture of the disease and an assessment of the efficacy of treatment.

\section{Gene Transcript PCR Analysis}

A gene biomarker assay that comprises a validated circulating 'fingerprint' of NETs has been developed. Individual genes were selected by analyzing microarray data sets of cellular profiles from fresh frozen tumors as well as from whole blood of patients with NETs to identify similarities in expression patterns. Once identified, undertaking coexpression network inference with normal tissue eliminated genes that were considered unlikely to be malignant [6]. A similar strategy was used to minimize neoplasia-associated genes from other tumor types (e.g. breast). Using this analytic and computational strategy, 
candidate marker genes detectable in peripheral circulation were identified as representative of NETs.

Redundancy of expressed genes and systematically selected analytes renders this type of test significantly more robust than monoanalyte tests such as CgA or pancreastatin [5]. The NET MAAA biomarker panel has been demonstrated to exhibit a reliably high level of sensitivity and specificity (98 and 97\%, respectively). The PCR test is standardized and reproducible (inter- and intra-assay coefficient of variation $<2 \%$ ), and is not affected by age, gender, ethnicity, fasting, or PPI medication [7]. As in the case of other MAAAs, this requires a dedicated laboratory since multigene array assessment necessitates a specialized analysis. Nonetheless, previous facsimiles of multianalyte deployment, including FibroSure and a variety of breast cancer tests, indicate that this is a viable model.

In terms of clinical benefit, apart from accurately confirming disease, the most immediate added value provided is in the assessment of treatment efficacy. The dimensionality of a multianalyte test offers considerable insight into the biological pathways exploited by neoplastic transformation as well as the availability of targets for therapy, and can provide information for assessment of response. This is best exemplified by results linking therapy e.g. somatostatin analogs to RECIST-measured responses [87]. This is in contrast to the monodimensionality of single analyte values, which are limited in their ability to provide any therapeutically relevant information other than estimates of 'relative' tumor burden or a general response to treatment.

\section{Future Advances}

It is indubitable that technological improvements in objective measurement (mutations, methylation, protein expression, molecular imaging) will drive the development of cancer detection biomarkers. These will include ultrahigh-throughput sequencing technology - whole genome sequencing of germ-line DNA - to identify rare, highly penetrant, high-risk alleles that can be used to focus cancer screening protocols on individuals at high risk. Similarly, the generation of robust sequencing protocols for use in small tissue samples or single cells will provide opportunities to investigate genomic changes early in the malignant process. Evaluation of early- and late-stage samples will be used to identify biomarkers associated with progressive disease. Similarly, it is likely that whole transcriptome sequencing will identify RNA splice variants and noncoding RNAs that can be used in cancer detection [88]. Ultimately, the use of high-level deep se- quencing of cell-free DNA in plasma or urine could also be used to identify tumor-derived DNA fragments with mutations, gene fusions, DNA methylation changes, or structural rearrangements that are pathognomonic for specific malignancies.

\section{Conclusions}

Given the complexity of the neoplastic process and the heterogeneity of NETs, as well as the tumor microenvironment, it is unlikely that any single diagnostic will be effective. Rather, diagnosis will be a staged process initiated by the identification of individuals at risk, followed by the performance of a targeted screen using an easily accessed biosample such as blood or urine, and finally by localization of the lesions with molecular imaging. Once localized, tumors can be biopsied to assess activity and select therapy. In this process biomarkers will represent a key facet of the gnosis of neoplasia and, by the very nature of their relationship with the disease, articulate our clinical relationship with it. The quantification and vernacular with which biomarkers are evaluated and judged, therefore, will delineate the understanding of the disease itself. In the past, singleanalyte biomarkers have been highly effective in identifying distinguishing features of different diseases. However, in NETs and many other cancers, the philology of single analytes no longer accurately describes the complexity of these diseases. The development of extensive data sets of measurements of marker genes comprising numerous individual tumors and patient profiles will need to be accumulated to create a portrait of different NET diseases over time. The development and use of multianalyte biomarkers represents an important initial step in the future of personalized medicine, with the potent combination of highyielding, multidimensional information and minimally invasive procedures for patients. This is especially true for NETs, whose protean clinical expression, often indolent course and late detection make them particularly unsuitable for the classic single-analyte approach. With a new, expansive genetic language to describe NETs, the development of sensitive and specific multianalyte biomarker tests combined with novel functional imaging strategies will provide a broader and more effective platform for management. Ultimately, better biomarkers will translate directly into better medical care at a lower cost. For a disease whose challenges are defined by its complexity and obscurity, the curative potential of MAAA biomarkers will be compounded by synergistic applications of early diagnosis, imaging and prognostic determinations. 


\section{References}

1 McDermott U, Downing JR, Stratton MR: Genomics and the continuum of cancer care. N Engl J Med 2011;364:340-350.

$>2$ Paez JG, Janne PA, Lee JC, Tracy S, Greulich H, Gabriel S, Herman P, Kaye FJ, Lindeman N, Boggon TJ, Naoki K, Sasaki H, Fujii Y, Eck MJ, Sellers WR, Johnson BE, Meyerson M: EGFR mutations in lung cancer: correlation with clinical response to gefitinib therapy. Science 2004;304:1497-1500.

3 Gayed I, Vu T, Iyer R, Johnson M, Macapinlac $\mathrm{H}$, Swanston N, Podoloff D: The role of ${ }^{18} \mathrm{~F}$ FDG PET in staging and early prediction of response to therapy of recurrent gastrointestinal stromal tumors. J Nucl Med 2004;45:1721.

-4 Bodei L, Sundin A, Kidd M, Prasad V, Modlin I: The status of neuroendocrine tumor imaging: from darkness to light? Neuroendocrinology 2014, Epub ahead of print.

$>5$ Modlin I, Drozdov I, Alaimo D, Callahan S, Teixeira N, Bodei L, Kidd M: A multianalyte PCR blood test outperforms single analyte ELISAs for neuroendocrine tumor detection. Endocr Relat Cancer 2014;21:615-628.

$\checkmark 6$ Modlin I, Drozdov I, Kidd M: The identification of gut neuroendocrine tumor disease by multiple synchronous transcript analysis in blood. PLoS One 2013;8:e63364.

7 Modlin I, Drozdov I, Kidd M: Gut neuroendocrine tumor blood qPCR fingerprint assay: characteristics and reproducibility. Clin Chem 2014;52:419-429.

8 Hulka B: Overview of biological markers; in Hulka B, Griffith J, Wilcosky T (eds): Biological Markers in Epidemiology. New York, Oxford University Press, 1990, pp 3-15.

9 Frank R, Hargreaves R: Clinical biomarkers in drug discovery and development. Nat Rev Drug Discov 2003;2:566-580.

$>10$ Hanahan D, Weinberg RA: Hallmarks of cancer: the next generation. Cell 2011;144:646674.

-11 Garcia-Closas M, Hall P, Nevanlinna H, et al: Heterogeneity of breast cancer associations with five susceptibility loci by clinical and pathological characteristics. PLoS Genet 2008; 4:e1000054.

12 Kiemeney LA, Thorlacius S, Sulem P, et al: Sequence variant on $8 \mathrm{q} 24$ confers susceptibility to urinary bladder cancer. Nat Genet 2008;40: 1307-1312.

$>13$ Song H, Ramus SJ, Tyrer J, et al: A genomewide association study identifies a new ovarian cancer susceptibility locus on 9p22.2. Nat Genet 2009;41:996-1000.

14 Cancer Genome Atlas Research Network: Integrated genomic analyses of ovarian carcinoma. Nature 2011;474:609-615.

$>15$ Dong SM, Traverso G, Johnson C, Geng L, Favis R, Boynton K, Hibi K, Goodman SN, D’Allessio M, Paty P, Hamilton SR, Sidransky D, Barany F, Levin B, Shuber A, Kinzler KW, Vogelstein B, Jen J: Detecting colorectal cancer in stool with the use of multiple ge- netic targets. J Natl Cancer Inst 2001;93:858865.

16 Hoque MO, Begum S, Topaloglu O, Jeronimo C, Mambo E, Westra WH, Califano JA, Sidransky D: Quantitative detection of promoter hypermethylation of multiple genes in the tumor, urine, and serum DNA of patients with renal cancer. Cancer Res 2004;64:55115517.

17 Hoque MO, Feng Q, Toure P, Dem A, Critchlow CW, Hawes SE, Wood T, Jeronimo C, Rosenbaum E, Stern J, Yu M, Trink B, Kiviat NB, Sidransky D: Detection of aberrant methylation of four genes in plasma DNA for the detection of breast cancer. J Clin Oncol 2006;24:4262-4269.

18 Topaloglu O, Hoque MO, Tokumaru Y, Lee J, Ratovitski E, Sidransky D, Moon CS: Detection of promoter hypermethylation of multiple genes in the tumor and bronchoalveolar lavage of patients with lung cancer. Clin Cancer Res 2004;10:2284-2288.

19 Chen J, Rocken C, Lofton-Day C, Schulz HU, Muller O, Kutzner N, Malfertheiner P, Ebert MP: Molecular analysis of APC promoter methylation and protein expression in colorectal cancer metastasis. Carcinogenesis 2005;26:37-43.

20 Salami SS, Schmidt F, Laxman B, Regan MM, Rickman DS, Scherr D, Bueti G, Siddiqui J, Tomlins SA, Wei JT, Chinnaiyan AM, Rubin MA, Sanda MG: Combining urinary detection of TMPRSS2: ERG and PCA3 with serum PSA to predict diagnosis of prostate cancer. Urol Oncol 2013;31:566-571.

21 Lewis MA, Yao JC: Molecular pathology and genetics of gastrointestinal neuroendocrine tumours. Curr Opin Endocrinol Diabetes Obes 2013;4:4.

22 Chaffer CL, Weinberg RA: A perspective on cancer cell metastasis. Science 2011;331: 1559-1564.

23 Reya T, Morrison SJ, Clarke MF, Weissman IL: Stem cells, cancer, and cancer stem cells. Nature 2001;414:105-111.

24 Stephens PJ, Greenman CD, Fu B, et al: Massive genomic rearrangement acquired in a single catastrophic event during cancer development. Cell 2011;144:27-40.

25 Lawrence B, Gustafsson BI, Chan A, Svejda B, Kidd M, Modlin IM: The epidemiology of gastroenteropancreatic neuroendocrine tumors. Endocrinol Metab Clin North Am 2011;40:1-18, vii.

26 Pepe MS: An interpretation for the roc curve and inference using GLM procedures. Biometrics 2000;56:352-359.

27 Shapiro DE: The interpretation of diagnostic tests. Stat Methods Med Res 1999;8:113-134.

28 Parikh R, Mathai A, Parikh S, Chandra Sekhar G, Thomas R: Understanding and using sensitivity, specificity and predictive values. Indian J Ophthalmol 2008;56:45-50.

29 Tsikitis VL, Wertheim BC, Guerrero MA: Trends of incidence and survival of gastroin- testinal neuroendocrine tumors in the united states: a seer analysis. J Cancer 2012;3:292302.

30 Duque M, Modlin IM, Gupta A, Saif MW: Biomarkers in neuroendocrine tumors. JOP 2013;14:372-376.

31 Stridsberg M, Eriksson B, Oberg K, Janson ET: A comparison between three commercial kits for chromogranin a measurements. J Endocrinol 2003;177:337-341.

32 Reid CN, Stevenson M, Abogunrin F, Ruddock MW, Emmert-Streib F, Lamont JV, Williamson KE: Standardization of diagnostic biomarker concentrations in urine: the hematuria caveat. PLoS One 2012;7:e53354.

-33 Poynard T, Imbert-Bismut F, Munteanu M, Messous D, Myers RP, Thabut D, Ratziu V, Mercadier A, Benhamou Y, Hainque B: Overview of the diagnostic value of biochemical markers of liver fibrosis (FibroTest, HCV FibroSure) and necrosis (ActiTest) in patients with chronic hepatitis C. Comp Hepatol 2004;3:8.

34 Poynard T, Ngo Y, Munteanu M, Thabut D, Ratziu V: Noninvasive markers of hepatic fibrosis in chronic hepatitis B. Curr Hepat Rep 2011;10:87-97.

35 Sang M, Wu X, Fan X, Sang M, Zhou X, Zhou $\mathrm{N}$ : Multiple MAGE-A genes as surveillance marker for the detection of circulating tumor cells in patients with ovarian cancer. Biomarkers 2014;19:34-42.

36 Chadeau-Hyam M, Vermeulen RC, Hebels DG, Castagne R, Campanella G, Portengen L, Kelly RS, Bergdahl IA, Melin B, Hallmans G, Palli D, Krogh V, Tumino R, Sacerdote C, Panico S, de Kok TM, Smith MT, Kleinjans JC, Vineis P, Kyrtopoulos SA: Prediagnostic transcriptomic markers of chronic lymphocytic leukemia reveal perturbations 10 years before diagnosis. Ann Oncol 2014;25:10651072.

37 Firestein GS: A biomarker by any other name. Nat Clin Pract Rheumatol 2006;2:635635.

38 Modlin IM, Gustafsson BI, Moss SF, Pavel M, Tsolakis AV, Kidd M: Chromogranin A - biological function and clinical utility in neuro endocrine tumor disease. Ann Surg Oncol 2010;17:2427-2443.

39 Marotta V, Nuzzo V, Ferrara T, Zuccoli A, Masone M, Nocerino L, Del Prete M, Marciello F, Ramundo V, Lombardi G, Vitale M, Colao A, Faggiano A: Limitations of chromogranin A in clinical practice. Biomarkers 2012;17:186-191.

40 Modlin IM, Oberg K, Chung DC, Jensen RT, de Herder WW, Thakker RV, Caplin M, Delle Fave G, Kaltsas GA, Krenning EP, Moss SF, Nilsson O, Rindi G, Salazar R, Ruszniewski P, Sundin A: Gastroenteropancreatic neuroendocrine tumours. Lancet Oncol 2008;9:61-72.

-41 Modlin IM, Latich I, Zikusoka M, Kidd M, Eick G, Chan AK: Gastrointestinal carcinoids: the evolution of diagnostic strategies. J Clin Gastroenterol 2006;40:572-582. 
-42 Kanakis G, Kaltsas G: Biochemical markers for gastroenteropancreatic neuroendocrine tumours (GEP-NETs). Best Pract Res Clin Gastroenterol 2012;26:791-802.

-43 Wu JT, Erickson AJ, Tsao KC, Wu TL, Sun CF: Elevated serum chromogranin A is detectable in patients with carcinomas at advanced disease stages. Ann Clin Lab Sci 2000; 30:175-178.

-44 Sciarra A, Monti S, Gentile V, Salciccia S, Gomez AM, Pannunzi LP, Di Silverio F: Chromogranin A expression in familial versus sporadic prostate cancer. Urology 2005;66:10101014.

45 Hsiao RJ, Mezger MS, O’Connor DT: Chromogranin A in uremia: progressive retention of immunoreactive fragments. Kidney Int 1990;37:955-964.

-46 Jianu CS, Fossmark R, Syversen U, Hauso O, Waldum HL: A meal test improves the specificity of chromogranin A as a marker of neuroendocrine neoplasia. Tumour Biol 2010;31: 373-380.

-47 Lawrence B, Gustafsson BI, Kidd M, Pavel M, Svejda B, Modlin IM: The clinical relevance of chromogranin A as a biomarker for gastroenteropancreatic neuroendocrine tumors. Endocrinol Metab Clin North Am 2011;40:111134.

48 de Souto Barreto P, Lapeyre-Mestre M, Mathieu C, Piau C, Bouget C, Cayla F, Vellas B, Rolland Y: Prevalence and associations of the use of proton pump inhibitors in nursing homes: a cross-sectional study. J Am Med Dir Assoc 2013;14:265-269.

49 Gerson LB, Kahrilas PJ, Fass R: Insights into gastroesophageal reflux disease-associated dyspeptic symptoms. Clin Gastroenterol Hepatol 2011;9:824-833.

50 Campana D, Nori F, Piscitelli L, Morselli-Labate AM, Pezzilli R, Corinaldesi R, Tomassetti P: Chromogranin A: is it a useful marker of neuroendocrine tumors? J Clin Oncol 2007;25:1967-1973.

-51 Zatelli MC, Torta M, Leon A, Ambrosio MR, Gion M, Tomassetti P, De Braud F, Delle Fave G, Dogliotti L, degli Uberti EC; Italian CromaNet Working Group: Chromogranin $\mathrm{A}$ as a marker of neuroendocrine neoplasia: an Italian Multicenter Study. Endocr Relat Cancer 2007;14:473-482.

-52 Jensen KH, Hilsted L, Jensen C, Mynster T, Rehfeld JF, Knigge U: Chromogranin A is a sensitive marker of progression or regression in ileo-cecal neuroendocrine tumors. Scand J Gastroenterol 2013;48:70-77.

-53 Stridsberg M, Oberg K, Li Q, Engstrom U, Lundqvist G: Measurements of chromogranin A, chromogranin B (secretogranin I), chromogranin C (secretogranin II) and pancreastatin in plasma and urine from patients with carcinoid tumours and endocrine pancreatic tumours. J Endocrinol 1995;144:4959.

54 Calhoun K, Toth-Fejel S, Cheek J, Pommier $\mathrm{R}$ : Serum peptide profiles in patients with carcinoid tumors. Am J Surg 2003;186:28-31.
55 Raines D, Chester M, Diebold AE, Mamikunian P, Anthony CT, Mamikunian G, Woltering EA: A prospective evaluation of the effect of chronic proton pump inhibitor use on plasma biomarker levels in humans. Pancreas 2012;41:508-511.

56 Ito T, Igarashi H, Jensen RT: Serum pancreastatin: the long sought for universal, sensitive, specific tumor marker for neuroendocrine tumors (NETs)? Pancreas 2012;41:505507.

57 Turner GB, Johnston BT, McCance DR, McGinty A, Watson RGP, Patterson CC, Ardill JE: Circulating markers of prognosis and response to treatment in patients with midgut carcinoid tumours. Gut 2006;55:15861591.

-58 Siegel EG, Gallwitz B, Folsch UR, Schmidt WE: Effect of human pancreastatin peptide (hP-16) on oral glucose tolerance in man. Exp Clin Endocrinol Diabetes 1998;106: 178-182.

-59 Valicherla GR, Hossain Z, Mahata SK, Gayen JR: Pancreastatin is an endogenous peptide that regulates glucose homeostasis. Physiol Genomics 2013;45:1060-1071.

60 Bergenfelz A, Luts L, Jensen TB, Sundler F: Pancreastatin plasma levels in patients with primary hyperparathyroidism. World J Surg 2000;24:1579-1583.

61 O'Dorisio TM, Krutzik SR, Woltering EA, Lindholm E, Joseph S, Gandolfi AE, Wang YZ, Boudreaux JP, Vinik AI, Go VL, Howe JR, Halfdanarson T, O'Dorisio MS, Mamikunian G: Development of a highly sensitive and specific carboxy-terminal human pancreastatin assay to monitor neuroendocrine tumor behavior. Pancreas 2010;39:611-616.

-62 Bech PR, Martin NM, Ramachandran R, Bloom SR: The biochemical utility of chromogranin A, chromogranin B and cocaineand amphetamine-regulated transcript for neuroendocrine neoplasia. Ann Clin Biochem 2014;51:8-21.

63 Baudin E, Gigliotti A, Ducreux M, Ropers J, Comoy E, Sabourin JC, Bidart JM, Cailleux AF, Bonacci R, Ruffié P, Schlumberger M: Neuron-specific enolase and chromogranin $\mathrm{A}$ as markers of neuroendocrine tumours. $\mathrm{Br}$ J Cancer 1998;78:1102-1107.

-64 Tellez MR, Mamikunian G, O’Dorisio TM, Vinik AI, Woltering EA: A single fasting plasma 5-HIAA value correlates with 24-hour urinary 5-HIAA values and other biomarkers in midgut neuroendocrine tumors (NETs). Pancreas 2013;42:405-410.

65 Bajetta E, Ferrari L, Martinetti A, Celio L, Procopio G, Artale S, Zilembo N, Di Bartolomeo M, Seregni E, Bombardieri E: Chromogranin A, neuron-specific enolase, carcinoembryonic antigen, and hydroxyindole acetic acid evaluation in patients with neuroendocrine tumors. Cancer 1999;86:858-865.

66 Dobson R, Burgess MI, Banks M, Pritchard DM, Vora J, Valle JW, Wong C, Chadwick C, George K, Keevil B, Adaway J, Ardill JES, Anthoney A, Hofmann U, Poston GJ, Cuthbert- son DJ: The association of a panel of biomarkers with the presence and severity of carcinoid heart disease: a cross-sectional study. PLoS One 2013;8:e73679.

67 Feldman JM, O’Dorisio TM: Role of neuropeptides and serotonin in the diagnosis of carcinoid tumors. Am J Med 1986;81:41-48.

68 Eriksson B, Oberg K, Stridsberg M: Tumor markers in neuroendocrine tumors. Digestion 2000;62(suppl 1):33-38.

69 Langstein HN, Norton JA, Chiang V, O'Dorisio TM, Maton PN, Marx SJ, Jensen RT: The utility of circulating levels of human pancreatic polypeptide as a marker for islet cell tumors. Surgery 1990;108:1109-1115, discussion 1115-1116.

70 Walter T, Chardon L, Chopin-laly X, Raverot V, Caffin A-G, Chayvialle J-A, Scoazec J-Y, Lombard-Bohas $\mathrm{C}$ : Is the combination of chromogranin A and pancreatic polypeptide serum determinations of interest in the diagnosis and follow-up of gastro-entero-pancreatic neuroendocrine tumours? Eur J Cancer 2012;48:1766-1773.

71 Korse CM, Taal BG, Groot CAd, Bakker RH, Bonfrer JMG: Chromogranin A and N-terminal pro-brain natriuretic peptide: an excellent pair of biomarkers for diagnostics in patients with neuroendocrine tumor. J Clin Oncol 2009;27:4293-4299.

72 Behnes M, Brueckmann M, Lang S, Weiss C, Ahmad-Nejad P, Neumaier M, Borggrefe M, Hoffmann U: Connective tissue growth factor (CTGF/CCN2): diagnostic and prognostic value in acute heart failure. Clin Res Cardiol 2014;103:107-116.

73 Bergestuen DS, Gravning J, Haugaa KH, Sahakyan LG, Aakhus S, Thiis-Evensen E, Oie E, Aukrust P, Attramadal H, Edvardsen T: Plasma CCN2/connective tissue growth factor is associated with right ventricular dysfunction in patients with neuroendocrine tumors. BMC Cancer 2010;10:6.

-74 Korse CM, Taal BG, Bonfrer JM, Vincent A, van Velthuysen ML, Baas P: An elevated progastrin-releasing peptide level in patients with well-differentiated neuroendocrine tumours indicates a primary tumour in the lung and predicts a shorter survival. Ann Oncol 2011; 22:2625-2630.

75 Khan MS, Tsigani T, Rashid M, Rabouhans JS, Yu D, Luong TV, Caplin M, Meyer T: Circulating tumor cells and EpCAM expression in neuroendocrine tumors. Clin Cancer Res 2011;17: 337-345.

76 Khan MS, Kirkwood A, Tsigani T, GarciaHernandez J, Hartley JA, Caplin ME, Meyer $\mathrm{T}$ : Circulating tumor cells as prognostic markers in neuroendocrine tumors. J Clin Oncol 2013;31:365-372.

77 Cristofanilli M, Hayes DF, Budd GT, Ellis MJ, Stopeck A, Reuben JM, Doyle GV, Matera J, Allard WJ, Miller MC, Fritsche HA, Hortobagyi GN, Terstappen LW: Circulating tumor cells: a novel prognostic factor for newly diagnosed metastatic breast cancer. J Clin Oncol 2005;23:1420-1430. 
78 Cohen SJ, Punt CJ, Iannotti N, Saidman BH, Sabbath KD, Gabrail NY, Picus J, Morse M, Mitchell E, Miller MC, Doyle GV, Tissing H, Terstappen LW, Meropol NJ: Relationship of circulating tumor cells to tumor response, progression-free survival, and overall survival in patients with metastatic colorectal cancer. J Clin Oncol 2008;26:3213-3221.

79 Resel Folkersma L, San Jose Manso L, Galante Romo I, Moreno Sierra J, Olivier Gomez C: Prognostic significance of circulating tumor cell count in patients with metastatic hormone-sensitive prostate cancer. Urology 2012;80:1328-1332.

80 Di Leva G, Croce CM: miRNA profiling of cancer. Curr Opin Genet Dev 2013;23:3-11.

-81 Roldo C, Missiaglia E, Hagan JP, Falconi M, Capelli P, Bersani S, Calin GA, Volinia S, Liu CG, Scarpa A, Croce CM: MicroRNA expression abnormalities in pancreatic endocrine and acinar tumors are associated with distinctive pathologic features and clinical behavior. J Clin Oncol 2006;24:46774684 .
82 Thorns C, Schurmann C, Gebauer N, Wallaschofski H, Kumpers C, Bernard V, Feller AC, Keck T, Habermann JK, Begum N, Lehnert H, Brabant G: Global microRNA profiling of pancreatic neuroendocrine neoplasias. Anticancer Res 2014;34:2249-2254.

83 Li A, Yu J, Kim H, Wolfgang CL, Canto MI, Hruban RH, Goggins M: MicroRNA array analysis finds elevated serum miR-1290 accurately distinguishes patients with low-stage pancreatic cancer from healthy and disease controls. Clin Cancer Res 2013;19:36003610 .

84 Li SC, Essaghir A, Martijn C, Lloyd RV, Demoulin JB, Oberg K, Giandomenico V: Global microRNA profiling of well-differentiated small intestinal neuroendocrine tumors. Mod Pathol 2013;26:685-696.

85 Gounden V, Zhao Z: MicroRNAs as potential biomarkers: is the future here? Clin Physiol Biochem - Scientific Shorts.

86 Modlin IM, Moss SF, Chung DC, Jensen RT, Snyderwine E: Priorities for improving the management of gastroenteropancreatic neuroendocrine tumors. J Natl Cancer Inst 2008; 100:1282-1289

87 Modlin I, Drozdov I, Kidd M: A multitranscript blood neuroendocrine tumor molecular signature to identify treatment efficacy and disease progress. J Clin Oncol 2013; 31(suppl):A4137.
88 Prensner JR, Chinnaiyan AM: The emergence of lncRNAs in cancer biology. Cancer Discov 2011;1:391-407.

89 de Laat JM, Pieterman CR, Weijmans M, Hermus AR, Dekkers OM, de Herder WW, van der Horst-Schrivers AN, Drent ML, Bisschop PH, Havekes B, Vriens MR, Valk GD: Low accuracy of tumor markers for diagnosing pancreatic neuroendocrine tumors in multiple endocrine neoplasia type 1 patients. J Clin Endocrinol Metab 2013;98:4143-4151.

90 Tohmola N, Itkonen O, Sane T, Markkanen H, Joenvaara S, Renkonen R, Hamalainen E: Analytical and preanalytical validation of a new mass spectrometric serum 5-hydroxyindoleacetic acid assay as neuroendocrine tumor marker. Clin Chim Acta 2013;428C:38-43.

91 Rustagi S, Warner RR, Divino CM: Serum pancreastatin: the next predictive neuroendocrine tumor marker. J Surg Oncol 2013;108: 126-128.

92 Metz DC, Jensen RT: Gastrointestinal neuroendocrine tumors: pancreatic endocrine tumors. Gastroenterology 2008;135:1469-1492. 